\title{
Bound State Solution of Dirac Equation for Generalized Pöschl-Teller plus Trigomometric Pöschl-Teller Non- Central Potential Using SUSY Quantum Mechanics
}

\author{
Suparmi \& Cari \\ Physics Department, Sebelas Maret University \\ Jalan Ir. Sutami 36 A, Surakarta 57126 \\ Email: suparmi@uns.ac.id.
}

\begin{abstract}
The bound state solution of the Dirac equation for generalized PöschlTeller and trigonometric Pöschl-Teller non-central potentials was obtained using SUSY quantum mechanics and the idea of shape invariance potential. The approximate relativistic energy spectrum was expressed in the closed form. The radial and polar wave functions were obtained using raising and lowering of radial and polar operators. The orbital quantum numbers were found from the polar Dirac equation, which was solved using SUSY quantum mechanics and the idea of shape invariance.
\end{abstract}

Keywords: bound state solution; Dirac equation; generalized Pöschl-Teller potential; non-central potentials; SUSY quantum mechanics; trigonometric Pöschl-Teller potential.

\section{$1 \quad$ Introduction}

One of the important tasks of relativistic quantum mechanics is to find an accurate and exact solution of the Dirac equation for a certain potential. The bound state solutions of Dirac equations for some central or non-central physical potentials, which are a combination of the magnitude of repulsive vector potential $V(\vec{r})$ and attractive scalar potential $S(\vec{r})$, have been investigated intensively since they have important applications in quantum chemistry, nuclear physics, and high-energy physics. Dirac equations have been used to describe the motion of spin half particles governed by the strong force such that the relativistic effects are taken into account.

The Dirac equations for some physical central and non-central potentials have been investigated in the cases of spin and/or pseudo-spin symmetries by Alhaidari, Hu, et al., Hamzavi and Rajabi, Soylu, et al., Onate, et al., and Sukumar [1-6]. Spin symmetric and pseudo-spin symmetric concepts have been used to investigate the aspects of deformed nuclei in nuclear physics. The concept of spin symmetry has been applied to the spectra of meson and antinucleon by Ginocchio [7] and the pseudo-spin symmetry concept was used by

Received August $31^{\text {st }}, 2013$, Revised July $11^{\text {th }}, 2014$, Accepted for publication August $4^{\text {th }}, 2014$.

Copyright (C) 2014 Published by ITB Journal Publisher, ISSN: 2337-5760, DOI: 10.5614/j.math.fund.sci.2014.46.3.1 
Ginocchio and Madland [8] to explain the quasi-degeneracy of nucleon doublets, by Alberto [9] for exotic nuclei, by Troltenier for super-deformation in nuclei [10], and by Stuchbery [11] to establish an affective nuclear shellmodel scheme. They have been solved with the Nikiforov-Uvarof (NU) method by Zou, et al., Ikhdair and Sever, and Zhou, et al. [12-14], with the asymptotic iteration method by Soylu, et al., Debnath and Biswas [4,15], with SUSY quantum mechanics by Onate, et al. and Chen, et al. [5,16], Romanovski polynomials by Suparmi and Cari, Cari, et al., Suparmi, et al. [17-19], and via SUSY quantum mechanics with similarity transformation by Sukumar, Hall and Yesiltas $[6,20]$ to find the relativistic energy and the corresponding wave function.

Dirac equations for the case of exact spin symmetric occur when the difference between the magnitude of the repulsive vector potential with the attractive scalar potential is zero and the sum of the magnitude of the repulsive vector and attractive scalar potentials is equal to the given potential. The exact pseudo-spin symmetry occurs when the sum of the magnitude of the repulsive vector potential and the attractive scalar potential is zero and the difference between the vector with scalar potential is equal to the given potential, which is central or non-central.

The Dirac equations for non-central potentials that have been investigated mostly are a combination of radial shape invariant potentials, such as a Coulomb potential or a spherical or non-spherical harmonics oscillator with ring-shaped potentials by $\mathrm{Hu}$, et al., Ikhdair and Sever, Zhou, et al. [2,13-14]. Therefore it is worth investigating the Dirac equation for non-central potential for radial shape invariant potentials such as a radial generalized Pöschl-Teller potential. Non-central potentials are widely used in studying quantum chemistry such as the relativistic effect of the distorted nucleus or the interaction between ring-shaped molecules.

Dirac equations for non-central potentials are exactly solvable only for the swave. For the $l$-wave, the solvable systems are only Coulomb, spherical harmonics oscillator, Morse and Kratzer potentials, but other systems were only solved approximately by Ikhdair and Sever, Ikot and Akpabio, and Agboola $[13,21-22]$ due to the contribution of the centrifugal term, $\sim r^{-2}$. A suitable approximation scheme was conventionally proposed by Greene and Aldrich [23] that works well for hyperbolic/exponential and trigonometric potentials.

Up until now, to our knowledge, no work exists on the Dirac equation with noncentral potential that is a combination of a generalized Pöschl-Teller potential with a trigonometric Pöschl-Teller non-central potential. It was therefore, the priority purpose of the present work to give approximate analytic solutions of 
the Dirac equation for this potential using the SUSY quantum mechanics approach. This potential can be applied to study the relativistic effect of the complex vibration-rotation energy structure of multi-electron atoms.

In this paper we will attempt to solve the Dirac equation for a charged particle moving in a field governed by a generalized Pöschl-Teller potential, which is discussed in Derezinski and Wrochna [24] and, with simultaneous presence of a trigonometric Pöschl-Teller non-central potential, in Flugge [25], using super symmetric quantum mechanics (SUSY QM) with the idea of shape invariance in the case of exact spin symmetry. SUSY QM was developed based on Witten's proposal [26], while the idea of shape invariant potentials was proposed by Gendenshtein [27]. SUSY QM is a powerful tool to determine the energy spectrum and wave function of a class of shape invariant potentials as in Sukumar, Dutt, et al., and Gangopadhyaya [6, 28-30].

The relativistic energy spectrum and wave functions are obtainable by SUSY QM and the idea of shape invariance, because the Dirac equation for noncentral shape invariance is separable and in the case of exact spin symmetry or pseudo-spin symmetry reduces to a one-dimensional Schrödinger-like equation with shape invariant potential. The relativistic energy spectrum is obtained by using SUSY QM and the idea of shape invariance, while the wave functions are obtained by using lowering and raising SUSY operators as discussed in Dutt, et $a l$. and Gangopadhyaya [28-30]. SUSY QM and similarity transformation have also been used to determine the relativistic energy of simple central potentials by diagonalizing a pair of matrices of the upper and lower components of Dirac equations by Sukumar, Hall and Yesiltas [6, 20]. The generalized Pöschl-Teller potential is also called the hyperbolic Scarf II potential, as in Derezinski and Wrochna [24]. Some hyperbolic and trigonometric non-central potentials are exactly solvable within the approximation of the centrifugal term and their bound state solutions have been reported in previous papers by Cari and Suparmi, Suparmi, et al., Saregar, et al., and Ikdhair [31-37].

This paper is organized as follows. A brief review of SUSY QM is presented in Section 2. Solutions of radial and polar Dirac equations are presented in Section 3.1 and 3.2. The conclusion is presented in Section 4. 


\section{Review of the SUSY Quantum Mechanics Approach Using Operator and Shape Invariance}

\subsection{SUSY Quantum Mechanics}

According to the definition proposed by Witten [26], in a SUSY quantum system there are super charge operators $Q_{i}$ that commute with the Hamiltonian $H_{s S}$ and are given as

$$
\left[Q_{i}, H_{s}\right]=0 \text { with, } i=1,2,3, \ldots N
$$

They obey the anti commutation algebra

$$
\left\{Q_{i}, Q_{j}\right\}=\delta_{i} H_{s}
$$

with $H_{s s}$ called SUSY Hamiltonian. Witten proposed that SUSY QM is a onedimensional model of SUSY field theory and he stated that the simplest SUSY $\mathrm{QM}$ system has $\mathrm{N}=2$, where

$$
Q_{1}=\frac{1}{\sqrt{2}}\left(\sigma_{1} \frac{p}{\sqrt{2 m}}+\sigma_{2} \phi(x)\right) \text { and } Q_{2}=\frac{1}{\sqrt{2}}\left(\sigma_{2} \frac{p}{\sqrt{2 m}}+\sigma_{1} \phi(x)\right)
$$

where $\sigma_{i}$ are the usual Pauli spin matrices, $p=-i \hbar \frac{\partial}{\partial x}$ is the usual momentum operator, and $\phi(x)$ is the super-potential. By inserting Eq. (3) into Eq. (2) we get,

$$
\begin{aligned}
& H_{s s}=\left(\begin{array}{cc}
-\frac{\hbar^{2}}{2 m} \frac{d^{2}}{d x^{2}}+\phi^{2}(x)+\frac{\hbar}{\sqrt{2 m}} \phi^{\prime}(x) & 0 \\
0 & -\frac{\hbar^{2}}{2 m} \frac{d^{2}}{d x^{2}}+\phi^{2}(x)-\frac{\hbar}{\sqrt{2 m}} \phi^{\prime}(x)
\end{array}\right) \text { (4) } \\
& =\left(\begin{array}{cc}
H_{+} & 0 \\
0 & H_{-}
\end{array}\right)
\end{aligned}
$$

with $H_{-}=-\frac{\hbar^{2}}{2 m} \frac{d^{2}}{d x^{2}}+V_{-}(x)$ for $V_{-}(x)=\phi^{2}(x)-\frac{\hbar}{\sqrt{2 m}} \phi^{\prime}(x)$

and $H_{+}=-\frac{\hbar^{2}}{2 m} \frac{d^{2}}{d x^{2}}+V_{+}(x)$ for $V_{+}(x)=\phi^{2}(x)+\frac{\hbar}{\sqrt{2 m}} \phi^{\prime}(x)$

Here $H_{-}$and $H_{+}$, are SUSY partners of the Hamiltonians, $V_{-}(x)$ and $V_{+}(x)$ are the SUSY partner potentials. To simplify the determination of the energy spectra and the wave functions, the new operators are introduced as 


$$
A^{+}=-\frac{\hbar}{\sqrt{2 m}} \frac{d}{d x}+\phi(x) \text { and } A=\frac{\hbar}{\sqrt{2 m}} \frac{d}{d x}+\phi(x)
$$

with $A^{+}$as raising operator, and $A$ as lowering operator. By manipulating Eqs. (5-7) we get

$$
H_{-}(x)=A^{+} A \text {, and } H_{+}(x)=A A^{+}
$$

It is always possible to factorize the usual Hamiltonian as

$$
H=H_{-}+E_{0}=\frac{\hbar^{2}}{2 m} \frac{d^{2}}{d x^{2}}+V_{-}\left(x, a_{0}\right)+E_{0}
$$

From Eqs. (5) and (9) we get,

$$
V(x)=V_{-}\left(x ; a_{0}\right)+E_{0}=\phi^{2}\left(x ; a_{0}\right)-\frac{\hbar}{\sqrt{2 m}} \phi^{\prime}\left(x ; a_{0}\right)+E_{0}
$$

where $V(x)$ is the effective potential, while $\phi(x)$, the super-potential, is determined hypothetically from Eq. (10), which is based on the shape of the effective potential of the system.

\subsection{Shape Invariance}

It is observed here that the SUSY only gives the relationship between the eigen values and eigen functions of the two Hamiltonian partners but does not yield the actual spectrum as discussed in Khare and Badhury [38]. In order to determine the energy spectrum, Gendenshtein introduced the shape invariance condition [27]. Accordingly, if the pair of SUSY partner potentials $V_{ \pm}(x)$ defined in Eqs. (5)-(6) are similar in shape and differ only in the parameters that appear in them, they are said to be shape invariant. More specifically, if $V_{ \pm}\left(x, a_{0}\right)$ satisfy the requirement that

$$
V_{+}\left(x, a_{j}\right)=V_{-}\left(x, a_{j+1}\right)+R\left(a_{j+1}\right)
$$

with

$$
\begin{aligned}
& V_{+}\left(x ; a_{j}\right)=\phi^{2}\left(x ; a_{j}\right)+\frac{\hbar}{\sqrt{2 m}} \phi^{\prime}\left(x ; a_{j}\right) \\
& V_{-}\left(x, a_{j+1}\right)=\phi^{2}\left(x, a_{j+1}\right)-\frac{\hbar}{\sqrt{2 m}} \phi\left(x ; a_{j+1}\right)
\end{aligned}
$$


where $j=0,1,2, . . ; a$ is a mapping parameter; $V_{-}\left(x, a_{0}\right)$ is associated with zero ground state energy; $a_{j}=f_{j}\left(a_{0}\right)$, where $f_{j}$ is a function applied $j$ times; the remainder, $R\left(a_{j}\right)$, is $a$ 's dependence but it is independent of $x$; then $V_{ \pm}\left(x, a_{0}\right)$ is said to be shape invariant. The energy eigen value of the Hamiltonian $H_{-}$is given by Gendenshtein [27] as follows

$$
E_{n}^{(-)}=\sum_{k=1}^{n} R\left(a_{k}\right)
$$

By using Eqs. (9) and (14) we get the total energy spectra,

$$
E_{n}=E_{n}^{(-)}+E_{0}
$$

Based on the characteristics of the lowering operator, the ground state wave function is obtained from the condition that

$$
A \psi_{0}^{(-)}=0
$$

Subsequently, the excited wave functions $\psi_{1}^{(-)}\left(x, a_{0}\right), \ldots \psi_{n}^{(-)}\left(x, a_{0}\right)$ of $H_{-}$ are obtained by using the raising operator operated on the lower wave function [36-38], given as

$$
y_{h}(5, q) \approx A(5, q) A(x q) A\left(5, q_{21}\right) y / 8(5, q)
$$

which is a generalization of the operator method for the one-dimensional harmonic oscillator potential. The energy spectra are obtained from Eqs. (10) and (14), while the wave function is obtained from Eqs. (16) and (17).

\section{Solution of Dirac Equation for Non-central Potential}

The Dirac equation with the scalar potential $S(\vec{r})$ and magnitude of vector potential $V(\vec{r})$ is given as in $\mathrm{Hu}$, et al. [2]

$$
\{\bar{\alpha} \cdot \vec{p}+\beta(M+S(\vec{r})\} \%(\vec{r})=\{E-V(\vec{r})\} \psi(\vec{r})
$$

where $M$ is the relativistic mass of the particle, $E$ is the total relativistic energy, and $\vec{p}$ is the three-dimensional momentum operator, $-i \nabla$

$$
\vec{\alpha}=\left(\begin{array}{cc}
0 & \vec{\sigma} \\
\vec{\sigma} & 0
\end{array}\right) \text {, and } \beta=\left(\begin{array}{cc}
I & 0 \\
0 & -I
\end{array}\right)
$$


with $\vec{\sigma}$ are the three-dimensional Pauli matrices and I is the $2 \times 2$ identity matrix. The potential in Eq. (18) is spherically symmetric potential, i.e. it does not only depend on the radial coordinate $r=|\vec{r}|$, and we have taken $\hbar=1, c=1$.

The Dirac equation expressed in Eq. (18) is invariant under spatial inversion and therefore its eigen states have definite parity. By writing the spinor as

$$
\psi(\vec{r})=\left(\begin{array}{l}
\zeta(\vec{r}) \\
\Omega(\vec{r})
\end{array}\right)=\left(\begin{array}{l}
\frac{F_{n K}(r)}{r} Y_{j n}^{l}(\theta, \varphi) \\
i \frac{G_{n K}(r)}{r} Y_{j n}^{I}(\theta, \varphi)
\end{array}\right)
$$

If we insert Eqs. (19) and (20) into Eq. (18) and use matrices multiplication, we achieve

$$
\begin{aligned}
& \bar{\sigma} \cdot \bar{p} \Omega(\bar{r})=\{-M-S(\bar{r})+E-V(\bar{r})\} \zeta(\bar{r})
\end{aligned}
$$

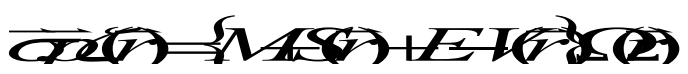

In the exact spin symmetric case, when the scalar potential is equal to the magnitude of vector potential $S(\vec{r})=V(\vec{r})$, then from Eqs. (21) and (22) we have

$$
\vec{\sigma} \cdot \vec{p} \frac{\vec{\sigma} \cdot \vec{p}}{M+E} \zeta(\vec{r})=\{-M-2 V(\vec{r})+E\} \zeta(\vec{r})
$$

By applying the Pauli matrices, it is simply shown that if $(\vec{a} \vec{p})(\vec{a} \vec{p})=p^{2}$, then Eq. (23) becomes

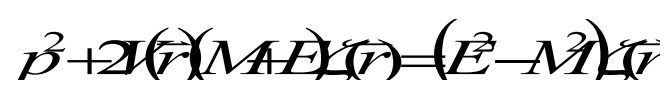

In the non-relativistic limit, where $E-M \rightarrow E_{N R}, E_{N R}$ is the non-relativistic energy and $E+M \rightarrow 2 \mu$, where $\mu$ is the non-relativistic mass, then Eq. (24) reduces to

$$
\left\{\frac{p^{2}}{2 \mu}+2 V(\vec{r})\right\} \zeta(\vec{r})=E_{N K} \zeta(\vec{r})
$$

Eq. (25) becomes the usual Schrödinger equation by setting $V(\vec{r}) \rightarrow \frac{V(\vec{r})}{2}$.

If the vector potential is non-central, i.e. a combination of generalized PöschlTeller potential and Pöschl-Teller non-central potential given as 


$$
V(r, \theta)=t^{2}\left(\frac{b^{2}+a(a+1)}{\operatorname{sinn}^{2} h r}-\frac{2 b\left(a+\frac{1}{2}\right) \cos h r}{\sin ^{2} h t r}\right)-\frac{1}{r^{2}}\left(\frac{\kappa(\kappa-1)}{\sin ^{2} \theta}+\frac{\eta(\eta-1)}{\cos ^{2} \theta}\right)
$$

then the Dirac equation for non-central potentials obtained from Eqs. (24) and (26) with $V(\vec{r}) \rightarrow \frac{V(r)}{2}$ is expressed as

$$
\begin{aligned}
& \left\{\frac{\partial}{\partial r}\left(r^{2} \frac{\partial}{\partial r}\right)+\frac{1}{\sin \theta} \frac{\partial}{\partial \theta}\left(\sin \theta \frac{\partial}{\partial \theta}\right)+\frac{1}{\sin ^{2} \theta} \frac{\partial^{2}}{\partial \varphi^{2}}\right\} \zeta(r, \theta, \varphi) \\
& -r^{2}(E+M) t^{2}\left(\frac{b^{2}+a(a+1)}{\sinh ^{2} t r}-\frac{2 b\left(a+\frac{1}{2} \cosh t r\right)}{\sinh ^{2} t r}\right) \zeta(r, \theta, \varphi) \\
& -(E+M)\left(\frac{\kappa(\kappa-1)}{\sin ^{2} \theta}+\frac{\eta(\eta-1)}{\cos ^{2} \theta}\right) \zeta(r, \theta, \varphi)=-r^{2}\left(E^{2}-M^{2}\right) \zeta(r, \theta, \varphi)
\end{aligned}
$$

where $0 \leq t r \leq \pi, \mathrm{b}>0, a>-\frac{1}{2}, \kappa>1, \eta>1,0 \leq \theta \leq \pi$, and in this case, $t>0$, the $t$ parameter has to control the width of the generalized Pöschl-Teller potential. Eq. (27) is solved using the variable separation method by setting $\zeta(r, \theta, \varphi)=\frac{\chi(r)}{r} P(\theta) \Phi(\varphi)$ and we have

$$
\begin{aligned}
& \frac{d^{2} \chi(r)}{d r^{2}}-\frac{l(l+1) \chi(r)}{r^{2}}-(E+M) t^{2}\left(\frac{b^{2}+a(a+1)}{\sinh ^{2} t r}-\frac{2 b\left(a+\frac{1}{2} \cosh t r\right)}{\sinh ^{2} t r}\right) \chi(r)+\left(E^{2}-M^{2}\right) \chi(r)=0 \\
& -\frac{1}{P \mathrm{~s} \mathrm{i} \theta} \frac{\partial}{\partial \theta}\left(\operatorname{si} \theta \frac{\partial P}{\partial \theta}\right)-\frac{1}{\Phi \mathrm{s} \mathrm{i}^{2} \theta} \frac{\partial^{2} \Phi}{\partial \varphi^{2}}+(E+M)\left(\frac{\kappa(\kappa-1)}{\mathrm{s} \mathrm{i}^{2} \theta}+\frac{\eta(\eta-1)}{\mathrm{c} \mathrm{o}^{2} \theta}\right)=l(l+1)
\end{aligned}
$$

\subsection{Solution of the Radial Dirac Equation}

In order to solve the radial Dirac equation in Eq. (28), we use the approximation value for the centrifugal term as in Greene and Aldirch, and in Ikdhair [13,23], 
$\frac{1}{r^{2}} \cong t^{2}\left(d_{0}+\frac{1}{\sinh ^{2} t r}\right)$, for $t r<<1$ and $d_{0}=1 / 12$. In the centrifugal approximation scheme, Eq. (28) becomes

$$
\begin{aligned}
& \frac{d^{2} \chi(r)}{d r^{2}}-t^{2}\left(\frac{\left(b^{2}+a(a+1)\right)(E+M)+l(l+1)}{\sinh t r}-\frac{2 b(E+M)\left(a+\frac{1}{2}\right) \cos h r}{\sinh t r}\right) \chi(r) \\
& +\left(E^{2}-M^{2}-t^{2} l(l+1) d_{0}\right) \chi(r)=0
\end{aligned}
$$

By setting

$$
\begin{aligned}
& (B+c(a+1))(E+M)(Z(l+1)=\in(c+1) \\
& 2 b\left(a+\frac{1}{2}\right)(E+M)=2 b\left(a+\frac{1}{2}\right), E^{2}-M^{2}-t^{2} l(l+1) d_{0}=\varepsilon
\end{aligned}
$$

Eq. (30) becomes

$$
-\frac{d^{2} \chi(r)}{d r^{2}}+t^{2}\left(\frac{c(c+1)}{\sinh t r}-\frac{2 b^{\prime}\left(a^{\prime}+\frac{1}{2}\right) \cosh r}{\sinh t r}\right) \chi(r)=\varepsilon \chi(r)
$$

and the effective potential in Eq. (33) is given as

$$
V=\frac{t^{2} c(c+1)}{\mathrm{s} \mathrm{i} \mathrm{n} \mathrm{n}^{2} t r}-\frac{t^{2} 2 b^{\prime}\left(a^{\prime}+\frac{1}{2}\right) \cos \mathbf{h}_{i}}{\mathrm{~s} \mathrm{i} \mathrm{n}^{2} \mathrm{lt} r}
$$

Eq. (33) is solved using SUSY QM and by introducing the hypothetical superpotential as in [26-27]

\section{(t) 1 Dot hat Btat}

By inserting Eqs. (34) and (35) into Eq. (10) we get

$$
\begin{aligned}
& \frac{t^{2} D^{2}}{\sinh t r}+t^{2} D^{2}+\frac{t^{2} B^{2}}{\sinh t r}+2 B D^{2} \frac{\cosh r}{\sinh t r}+\left(\frac{t^{2} D}{\sinh t r}+\frac{B t^{2} \cosh r}{\sinh t r}\right)= \\
& \frac{t^{2} c(c+1)}{\operatorname{sinhtr}}-\frac{2 t^{2} b^{\prime}\left(a^{\prime}+\frac{1}{2}\right) \cosh r}{\operatorname{sinhtr} r}-\varepsilon_{0}
\end{aligned}
$$

From Eq. (36) we have 


$$
\begin{aligned}
& \left(D^{2}+B^{2}+D\right)=(c(c+1)),(2 D B+B)=-2 b^{\prime}\left(a^{\prime}+\frac{1}{2}\right), \\
& \text { and } D^{2} t^{2}=-\varepsilon_{0}
\end{aligned}
$$

and thus from all expressions in Eq. (37) we get the values of D, B, and $\varepsilon_{0}$ that have physical meaning,

$$
\begin{aligned}
& D=\left(\sqrt{\frac{\left(c+\frac{1}{2}\right)^{2}-\sqrt{\left(\left(c+\frac{1}{2}\right)^{2}\right)^{2}-4\left(b^{\prime}\left(a^{\prime}+\frac{1}{2}\right)\right)^{2}}}{2}}-\frac{1}{2}\right) \\
& B=-\left(\sqrt{\frac{\left(c+\frac{1}{2}\right)^{2}+\sqrt{\left(\left(c+\frac{1}{2}\right)^{2}\right)^{2}-4\left(b^{\prime}\left(a^{\prime}+\frac{1}{2}\right)\right)^{2}}}{2}}\right)^{2} \\
& \varepsilon_{0}=-t^{2}\left(\sqrt{\frac{\left(c+\frac{1}{2}\right)^{2}-\sqrt{\left(\left(c+\frac{1}{2}\right)^{2}\right)^{2}-4\left(b^{\prime}\left(a^{\prime}+\frac{1}{2}\right)\right)^{2}}}{2}}-\frac{1}{2}\right)^{2}
\end{aligned}
$$

where Eq. (40) is the ground state relativistic energy equation of the system. By using Eqs. (35), (38) and (39), the super-potential is obtained, given as

$$
\phi(r)=t\left(N-\frac{1}{2}\right) \operatorname{coth} r-t K \operatorname{set} t
$$

with,

$$
\begin{aligned}
& N=\left(\sqrt{\frac{\left(c+\frac{1}{2}\right)^{2}-\sqrt{\left(\left(c+\frac{1}{2}\right)^{2}\right)^{2}-4\left(b^{\prime}\left(a^{\prime}+\frac{1}{2}\right)\right)^{2}}}{2}}\right) \\
& K=\sqrt{\frac{\left(c+\frac{1}{2}\right)^{2}+\sqrt{\left(c+\frac{1}{2}\right)^{4}-4\left(b^{\prime}\left(a^{\prime}+\frac{1}{2}\right)\right)^{2}}}{2}}
\end{aligned}
$$

By inserting Eq. (41) into Eqs. (5) and (6) we get the super-partner potentials as

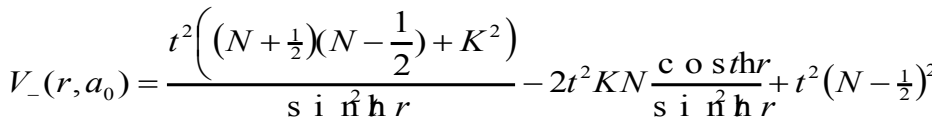

$$
\begin{aligned}
& V_{+}\left(r, a_{0}\right)=\frac{t^{2}\left(\left(N-\frac{1}{2}\right)\left(N-\frac{3}{2}\right)+K^{2}\right)}{\mathrm{s} \mathrm{i} \mathrm{h} h^{2} r}-K t^{2}(N-1) \frac{\mathrm{c} \mathrm{o} \mathrm{s} t h r}{\mathrm{~s} \mathrm{i} \mathrm{hh} r^{2} r}+t^{2}\left(N-\frac{1}{2}\right)^{\prime}
\end{aligned}
$$


By comparing the coefficient of the variables in Eqs. (44) and (45) we obtain the mapping parameters $a_{0}, a_{1}, \ldots a_{n}$ given as

$$
q_{b}=\mathrm{N}, q_{1}=\mathrm{N}+a_{n}=\mathrm{N} n
$$

By using Eqs. (44) and (46) we have

$$
V_{-}\left(r, a_{1}\right)=\frac{t^{2}\left(\left(N-\frac{1}{2}\right)\left(N-\frac{3}{2}\right)+K^{2}\right)}{\mathrm{s} \mathrm{i} \mathrm{h} \mathrm{h} r}-2 t^{2} K(N-1) \frac{\mathrm{c} \mathrm{o} \mathrm{s} t h r}{\mathrm{~s} \mathrm{i} \mathrm{h} \mathrm{h} r}+t^{2}\left(N-\frac{3}{2}\right)^{2}
$$

From Eqs. (45) and (47) it can be seen that $V_{+}\left(r, a_{0}\right)$ have the same function form as $V_{-}\left(r, a_{1}\right)$ and by using the shape invariance condition in Eq. (11), we get

$$
R\left(a_{1}\right)=V_{+}\left(r ; a_{0}\right)-V_{-}\left(r ; a_{1}\right)=t^{2}\left(\left(N-\frac{1}{2}\right)^{2}-\left(N-\frac{3}{2}\right)^{2}\right)
$$

By repeating the step used to determine $R\left(a_{1}\right)$ in Eq. (48) and the steps used to determine $V_{-}\left(r, a_{1}\right)$ in Eq. $(47)$ and by using Eqs. $(44-46,48)$ we obtain

$$
\begin{aligned}
& V_{-}\left(r, a_{2}\right)=\frac{t^{2}\left(\left(N-\frac{3}{2}\right)\left(N-\frac{5}{2}\right)+K^{2}\right)}{\sinh ^{2} t r}-2 t^{2} K((N-2)) \frac{\cosh t r}{\sinh ^{2} t r} \\
& +t^{2}\left(N-\frac{5}{2}\right)^{2} \\
& V_{+}\left(r, a_{1}\right)=\frac{t^{2}\left(\left(N-\frac{3}{2}\right)\left(N-\frac{5}{2}\right)+K^{2}\right)}{\sinh ^{2} t r}-2 t^{2} K((N-2)) \frac{\cosh t r}{\sinh ^{2} t r} \\
& +t^{2}\left(N-\frac{3}{2}\right)^{2}
\end{aligned}
$$

and so

$$
F\left(a_{2}\right)=V_{+}\left(r, q_{1}\right)-V\left(t, a_{2}\right)=I^{2}\left(\left(N_{2}\right)^{2}-\left(N_{2}\right)^{2}\right)
$$

Using generalizations of Eqs. (48) and (51) we obtain

$$
R\left(a_{n}\right)=V_{+}\left(r ; a_{n-1}\right)-V_{-}\left(r ; a_{n}\right)=t^{2}\left\{\left(N-\frac{1}{2}-(n-1)^{2}\right)-\left(N-\frac{1}{2}-n\right)^{2}\right\}
$$

Using Eqs. (14), (15) and (52) we get 


$$
\varepsilon_{n}^{(-)}=t^{2}\left\{\left(N-\frac{1}{2}\right)^{2}-\left(N-\frac{1}{2}-n\right)^{2}\right\} \text { that gives } \varepsilon_{n}=-t^{2}\left(N-\frac{1}{2}-n\right)^{2}
$$

The relativistic energy equation obtained from Eqs. (32) and (53) is

$$
E^{2}-M^{2}=-t^{2}\left\{\left(N-\frac{1}{2}-n\right)^{2}-l(l+1) d_{0}\right\}
$$

with

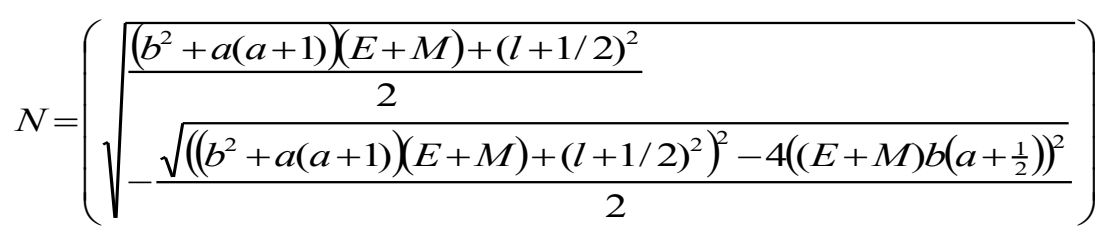

The relativistic energy spectrum is obtained numerically from the relativistic energy equation in Eq. (54) with the help of the Math-Lab software application.

In the non-relativistic limit, the relativistic energy reduces to non-relativistic energy as follows

$$
\begin{aligned}
& E^{2}-M^{2}=\left(E+M Y E-M Y=2 \mu E_{N}\right. \\
& \text { since }(E+M) \rightarrow 2 \mu \text { and }\left(E-M Y \rightarrow E_{N R}\right.
\end{aligned}
$$

If we set

$$
V=\frac{t^{2} c(c+1)}{\sinh ^{2} t r}-\frac{t^{2} 2 b^{\prime}\left(a^{\prime}+\frac{1}{2}\right) \cosh t r}{\sinh ^{2} t r} \rightarrow V=\frac{1}{2 \mu}\left\{\frac{t^{2} c(c+1)}{\sinh ^{2} t r}-\frac{t^{2} 2 b^{\prime}\left(a^{\prime}+\frac{1}{2}\right) \cosh t r}{\sinh ^{2} t r}\right\}
$$

then

$$
\left.\left.(E+M) B^{2}+d(a+1)\right) H(l+1)-\left(\frac{(E+M)}{2 \mu}\right) b^{2}+a(a+1)\right)+l(l+1)
$$

and

$$
2(E+M)\left(b\left(a+\frac{1}{2}\right)\right) \rightarrow 2 \frac{(E+M)\left(b\left(a+\frac{1}{2}\right)\right)}{2 \mu}
$$

Thus by applying Eqs. (56), (58) and (59) we obtain the value of $\mathrm{N}$ for $l=0$, given as 


$$
N=\sqrt{\frac{\sqrt{\frac{\left(2 \mu\left(b^{2}+a(a+1)\right)\right.}{2 \mu}+l(l+1)+\frac{1}{4}-\sqrt{\left(\frac{\left(2 \mu\left(b^{2}+a(a+1)\right)\right.}{2 \mu}+l(l+1)+\frac{1}{4}\right)^{2}}}}{2}}=a+\frac{1}{2}
$$

Therefore the non-relativistic energy obtained from Eqs. (54) and (60) is given as

$$
E_{N R}=-\frac{t^{2}}{2 \mu}\left\{(a-n)^{2}\right\}
$$

which is in agreement with the energy of the generalized Pöschl-Teller potential obtained using other methods [39].

By manipulating Eqs. (7), (16), (17), and (41) we obtain the ground state and first excited state wave function as follows. By inserting Eqs. (41) and (7) into Eq. (16), we get the radial ground state wave function as

$$
\left.\chi_{0}\left(a_{0}, r\right)=C(\sin h)\right)^{-(N-1 / 2)}(\operatorname{tant} \underset{2}{\operatorname{ta}})^{K}
$$

By manipulating Eqs. (7), (17), (41), and (62) we have

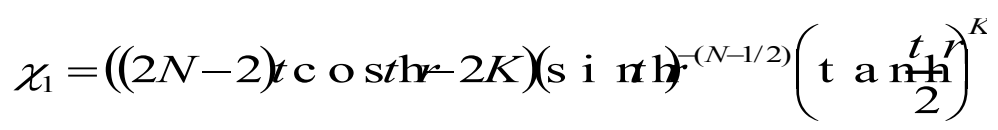

The second excited state wave function is obtained using Eqs. (5), (14) and (49), and so on for third, fourth, etc.

\subsection{Solution of the Angular Equation}

The solution of the polar Dirac equation is obtained by setting $P=\frac{Q}{\sqrt{\sin \theta}}$ in Eq. (29), so we have

$$
\frac{\partial^{2} Q}{\partial \theta^{2}}-\left\{\frac{(E+M) \kappa(\kappa-1)+m^{2}-\frac{1}{4}}{\sin ^{2} \theta}+\frac{(E+M) \eta(\eta-1)}{\cos \theta}\right\} Q+\left\{l(l+1)+\frac{1}{4}\right\} Q=0(64)
$$

To solve Eq. (64), we set 


$$
(E+M) \kappa(\kappa-1)+m^{2}-\frac{1}{4}=\kappa^{2}\left(\kappa^{2}-1\right)
$$

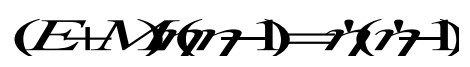

so Eq. (64) becomes a one-dimensional Dirac equation, given as

$$
\begin{aligned}
& \frac{\partial^{2} Q}{\partial \theta^{2}}+\left\{\frac{\pi^{2}\left(\pi^{2}-1\right)}{\mathrm{si}^{2} \phi}+\frac{\eta(\eta-1)}{\mathrm{co}^{2} S}\right\} Q=\left\{l(l+1)+\frac{1}{4}\right\} Q=E C \\
& V_{e}(\theta)=\frac{\kappa^{\prime}\left(\kappa^{\prime}-1\right)}{\mathrm{si} \mathrm{h} \theta}+\frac{\eta^{\prime}\left(\eta^{\prime}-1\right)}{\cos ^{2} \theta}
\end{aligned}
$$

where Eq. (68) shows the effective potential of the system. By considering Eqs. (10) and (68), the corresponding super-potential is introduced as

$$
\phi(Q)=D \text { a }
$$

By inserting Eq. (69) into Eq. (10) we have

$$
\frac{\kappa^{\prime}\left(\kappa^{\prime}-1\right)}{\mathrm{s} \mathrm{i}^{2} \boldsymbol{Q}}+\frac{\eta^{\prime}\left(\eta^{\prime}-1\right)}{\mathrm{c} \mathrm{o}^{2} \boldsymbol{\theta}}=\frac{D^{2}-D}{\mathrm{c} \mathrm{o}^{2} \boldsymbol{\theta}}+\frac{B^{2}+B}{\mathrm{~s} \mathrm{i}^{2} \boldsymbol{Q}}-B^{2}-D^{2}+2 D B+E_{\mathrm{o}}
$$

By comparing the coefficients of the variables on the left and right side in Eq. (70) we have the super-potential given as

$$
\phi(Q) \Rightarrow \lambda \text { aa ricot }
$$

and by setting the constant term on the right side equal to zero, we get

$$
E_{0}=\left(\eta+\kappa^{\prime}\right)^{2}
$$

By inserting Eq. (71) into Eqs. (5) and (6), we obtain the angular super-partner potential as

$$
\begin{aligned}
& V_{-}\left(a_{0}, \theta\right)=\frac{\kappa^{\prime}\left(\kappa^{\prime}-1\right)}{\mathrm{s} \mathrm{i}^{2} \mathrm{r} \theta}+\frac{\eta^{\prime}\left(\eta^{\prime}-1\right)}{\mathrm{co}^{2} \mathrm{~s} \theta}-\left(\eta^{\prime}+\kappa^{\prime}\right)^{2} \\
& V_{+}\left(a_{0}, \theta\right)=\frac{\kappa^{\prime}\left(\kappa^{\prime}+1\right)}{\mathrm{s} \mathrm{i}^{2}{ }^{2} \theta}+\frac{\eta^{\prime}\left(\eta^{\prime}+1\right)}{\mathrm{c} \mathrm{o}^{2} \mathrm{~s} \theta}-\left(\eta^{\prime}+\kappa^{\prime}\right)^{2}
\end{aligned}
$$

By shifting the parameter $\kappa^{\prime} \rightarrow \kappa^{\prime}+1$ and $\eta^{\prime} \rightarrow \eta^{\prime}+1$ in Eq. (73) and by applying Eq. (11) with Eqs. (73-74) we obtain the mapping parameters and $R\left(a_{1}\right)$ given as

$$
a_{0}=\kappa, a_{1}=\kappa+1, . a_{n}=\kappa+n ; \boldsymbol{b}_{\boldsymbol{B}}=\boldsymbol{n} \boldsymbol{b}=\boldsymbol{\eta}+\mathbf{1}, \ldots b_{r p}=\eta+n
$$

and 


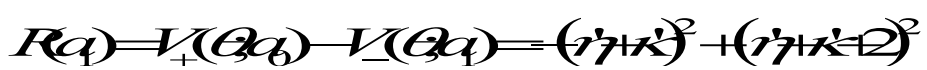

By shifting the parameter and $\kappa^{\prime} \rightarrow \kappa^{\prime}+2 \quad \eta^{\prime} \rightarrow \eta^{\prime}+2$ in Eq. (73) and $\kappa^{\prime} \rightarrow \kappa^{\prime}+1$ and $\eta^{\prime} \rightarrow \eta^{\prime}+1$ in Eq. (74) together with Eq. (11) we have

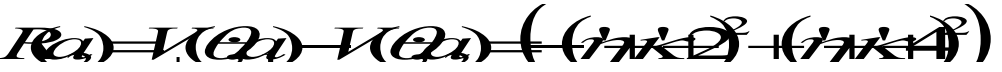

Thus by repeating the steps used in Eqs. (76) and (77), finally we get the general form of $\mathrm{R}$ given as

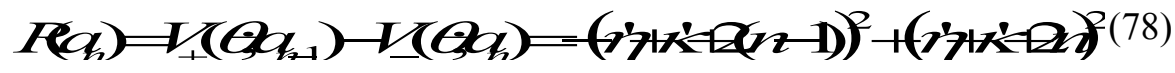

By manipulating Eqs. (14-15), (72), and (76-78) we have

$$
E_{n}^{(-)}=-\left(\eta+\kappa^{2}\right)^{2}+\left(\eta+\kappa^{2}+2 n\right)^{2} \text { and } E_{n}=\left(\eta+\kappa^{2}+2 n\right)^{2}
$$

and thus the orbital quantum number obtained from Eqs. (67) and (79) is give as

$$
\left\{l(l+1)+\frac{1}{4}\right\}=\left(\eta^{\prime}+\kappa^{\prime}+2 n\right)^{2}
$$

The value of $l$ that has physical meaning obtained from Eq. (80) is given as

$$
\left\{l+\frac{1}{2}\right\}=\sqrt{(E+M) \kappa(\kappa-1)+m^{2}}+\frac{1}{2}+\sqrt{(E+M) \prime h(\eta-1)+\frac{1}{4}}+\frac{1}{2}+2 n
$$

As in the radial part, the values of the orbital quantum number in the nonrelativistic limit are obtained from Eq. (81) as

$$
l=\sqrt{\left(\kappa(\kappa-1)+m^{2}\right.}+\eta+2 n
$$

This result is in agreement with the result obtained in Cari [39].

By using Eqs. (16) and (71) we get the relativistic polar ground state wave function as

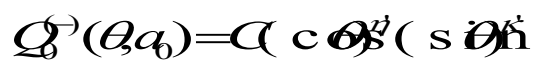

with

$$
\kappa^{\prime}=\sqrt{(E+M) \kappa(\kappa-1)+m^{2}}+\frac{1}{2} \text { and } \eta^{\prime}=\sqrt{(E+M) \eta(\eta-1)+\frac{1}{4}}+\frac{1}{2}
$$

By using Eqs. (7), (17), and (71), we find the first excited state wave function as

$$
Q^{\prime}(a, \theta)=\left\{( 2 \eta + 1 ) \operatorname { s i n } ^ { 2 } \theta ( 2 x ^ { 2 } + 1 ) \operatorname { c o s } ^ { 2 } \theta _ { j } \left(\operatorname { c o s } \theta ^ { \prime } \left(\sin \theta^{2}\right.\right.\right.
$$

The total ground state wave function is given as 


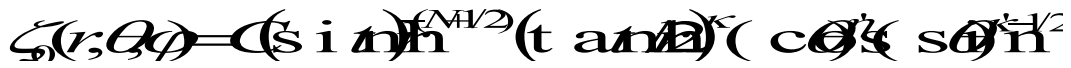

and the total first excited state wave function is obtained from combining Eqs. (63) and (85).

\section{Conclusion}

The Dirac equation with generalized Pöschl-Teller potential plus Pöschl-Teller non-central potential was solved using SUSY quantum mechanics because in the exact spin symmetric limit the radial and polar Dirac equations reduce to one-dimensional Schrödinger-like equations. In the approximation scheme of the centrifugal term, the super-potential, the raising and lowering operators and the mapping parameters were obtained from Dirac equations that have been reduced to Schrödinger-like equations. The relativistic energy equation was obtained exactly by using the super-potential and the idea of shape invariance with the radial generalized Pöschl-Teller potential as an effective potential, while the relativistic radial wave functions were obtained by using the lowering and raising operators.

In the non-relativistic limit, when the difference between relativistic energy $\mathrm{E}$ and particle mass $M$ is equal to the non-relativistic energy, while the sum of relativistic energy and relativistic mass is equal to twice the non-relativistic mass, the relativistic energy reduces to the non-relativistic energy of the generalized Pöschl-Teller potential with centrifugal distortion correction. For $l$ $=0$ we obtained the non-relativistic energy of the generalized Pöschl-Teller potential.

The relativistic polar wave function and relativistic orbital quantum number were found from the polar Dirac equation as in the radial part. The orbital quantum number was considered to be an energy variable in the Dirac equation. In the non-relativistic limit, the orbital quantum number reduces to the nonrelativistic quantum number and the angular wave functions, which are obtained by using the angular lowering and raising operators, reduce to non-relativistic angular wave functions.

\section{Acknowledgements}

This research was partly supported by a Higher Education Project Grant with contract no. 351/UN27.11/PN/2014. 


\section{References}

[1] Alhaidari, A.D., Relativistic Extension of Shape Invariant Potential, J. Phys. A, 34(46), pp. 9827- 9833, 2001.

[2] Hu, X.Q., Luo, G., Wu, Z.M., Niu, L.B., \& Ma, Y., Solving Dirac Equation with New Ring-Shaped Non-Spherical Harmonic Oscillator Potential, Commun. Theor. Phys., 53, pp. 242-249, 2010.

[3] Hamzavi, M. \& Rajabi, A.A., Spin and Pseudospin Symmetries with Trigonometric Pöschl-Teller Potential including Tensor Coupling, Adv. High En. Phys., 2013, pp. 1-12, 2013. ID 196986.

[4] Soylu, A., Bayrak, O. \& Boztosun, I., $\kappa$ State Solutions of the Dirac equation for the Eckart potential with pseudospin and spin symmetry, J. Phys. A: Math. Theor., 41, pp. 065308-1-8, 2008.

[5] Onate, C.A., Oyewumi, K.J. \& Falaye, B.J., An Approximate Solution of Dirac Equation for Second Pöschl-Teller like Scalar and Vector Potentials with a Coulomb Tensor Interaction, Af. Rev. Phys., 8(0020), pp. 129-137, 2013.

[6] Sukumar, C.V., Supersymmetry and the Dirac equation for a central Coulomb field, J. Phys. A: Math. Gen., 18, p. L697, 1985.

[7] Ginocchio, J.N., Relativistic Symmetries in Nuclei and Hadrons, Phys. Rep., 414, pp. 165-262, 2005.

[8] Ginocchio, J.N. \& Madland, D.G., Pseudospin Symmetry and Relativistic Single-Nucleon Wave Functions, Phys. Rev. C, 57(3), pp. 1167-1173, 1998.

[9] Alberto, P., Fiolhais, M., Malheiro, M., Delfino, A. \& Chiapparini, M., Pseudospinsymmetry as a Relativistic Dynamical Symmetry in the Nucleus, Phys. Rev. C, 65, pp. 034307-1-9, 2002.

[10] Troltenier, D., Nazarewicz, W., Szymanski, Z. \& Draayer, J.P., On the Validity of the Pseudo-Spin Concept for Axially Symmetric Deformed Nuclei, Nucl. Phys. A, 567, pp. 591-610, 1994.

[11] Stuchbery, A.E., Magnetic Properties of Rotational States in the PseudoNilsson Model, Nucl. Phys. A, 700, pp. 83-116, 2002.

[12] Zou, X., Yi, L.Y. \& Jia, C.S., Bound States of The Dirac Equation with Vector and Scalar Eckart Potentials, Phys. Lett. A, 346(1-3), pp. 54-64, 2005.

[13] Ikhdair, S.M. \& Sever, R., Exact Bound States of the D-dimensional Klein-Gordon Equation with Equal Scalar and Vector Ring-Shaped Pseudoharmonic Potential, Int. J. Mod. Phys. C, 19(9), pp.1425-1442, 2008.

[14] Zhou, F., Wu, Y. \& Guo, J.Y., Solutions of Dirac Equation for Makarov Potential with Pseudospin Symmetry, Commun. Theor. Phys. (Beijing, China), 52, pp. 813-816, 2009. 
[15] Debnath, S. \& Biswas, B., Analytical Solutions of the Klein-Gordon Equation for Rosen-Morse Potential via Asymptotic Iteration Method, EJTP, 9(26), 191-198, 2012.

[16] Chen, G., Chen, Z.D. \& Lou, Z.M., Bound State of Klein-Gordon and Dirac Equation for Scalar and Vector Pseudoharmonic Oscillator Potentials, Chin. Phys., 13(2), pp. 279-284, 2004.

[17] Suparmi, A. \& Cari, C., Solution of Dirac Equation for q-Deformed Eckart Potential with Yukawa-type Tensor Interaction for Spin and Pseudospin Symmetry Using Romanovski Polynomial, At. Ind. J., 39(3), pp. 112-123, 2013.

[18] Cari, C., Suparmi, A., Deta, U.A. \& Werdiningsih, I.S., Solution of Dirac Equation for Cotangent Potential with Coulomb-type Tensor Interaction for Spin and Pseudospin Symmetries Using Romanovski Polynomial, Makara. J. of Sci., 17(3), pp. 93-102, 2013.

[19] Suparmi, A., Cari, C. \& Deta, U.A., Exact Solution of Dirac equation for Scarf Potential with New Tensor Coupling Potential For Spin and Pseudospin Symmetries Using Romanovski Polynomials, Chin. Phys. B, 23(9), pp. 090304-1-10, 2014.

[20] Hall, R.L. \& Yesiltas, Ö., Supersymmetric Analysis for the Dirac Equation with Spin-Symmetric and Pseudo-Spin-Symmetric Interactions, arXiv:1006.4628v1 [math-ph], pp. 1-10, 2010.

[21] Ikot, A.N. \& Akpabio, L.E., Approximate Solution of the Schrodinger Equation with Rosen-Morse Potential Including the Centrifugal Term, App. Phys. Res., 2(2), pp. 202-208, 2010.

[22] Agboola, D., Dirac Equation with Spin Symmetry for the Modified Pöschl-Teller Potential in D Dimensions, Pramana, 76(6), pp. 875-885, 2011.

[23] Greene, R.I. \& Aldrich, C., Variational Wave Functions for a Screened Coulomb Potential, Phys. Rev. A, 14, pp. 2363, 1976.

[24] Derezinski, J. \& Wrochna, M., Exactly Solvable Schrödinger Operators, arXiv:1009.0541v2 [math-ph], 2010.

[25] Flugge, S., Practical Quantum Mechanics I, Springer, Berlin, 1994.

[26] Witten, E., Dynamical Breaking of Supersymmetry, Nucl. Phys. B, 188, pp. 513-554, 1981.

[27] Gendenshtein, L.E., Derivation of Exact Spectra of Schrodinger Equation by Means Supersymmetry, JETP Lett., 38, pp. 356-359, 1983.

[28] Dutt, R., Khare, A. \& Sukhatme, U.P., Supersymmetry, Shape Invariance and Exactly Solvable Potentials, Am. J. Phys., 56, pp. 163-168, 1988.

[29] Dutt, R., Gangopadhyaya, A. \& Sukhatme, U.P., Non-central potentials and Spherical Harmonics Using Supersymmetry and Shape Invariance, Am. J. Phys., 65(5), pp. 400-403, 1997.

[30] Gangopadhyaya, A., Mallow, J.V., Rasinariu, C. \& U.P. Sukhatme, Exact Solution of the Schrodinger Equation: Connection between 
Supersymmetric Quantum Mechanics and Spectrum Generating Algebras, Chin. J. Phys., 39(2), pp. 101-121, 2001.

[31] Suparmi, A., Cari, C., Handhika, J., Yanuarief, C. \& Marini, H., Approximate Solution of Schrodinger Equation for Modified PöschlTeller plus Trigonometric Rosen-Morse Non-Central Potentials in Terms of Finite Romanovski Polynomials, IOSR-JAP, 2(2), pp. 43-51, 2012.

[32] Cari, C. \& Suparmi, A., Approximate Solution of Schrodinger Equation for Trigonometric Scarf Potential with the Pöschl-Teller Non-central Potential Using NU Method, IOSR-JAP,2(3), pp. 13-23, 2012.

[33] Cari, C. \& Suparmi, A., Solution of Schrodinger Equation for Three Dimensional Harmonics Oscillator plus Rosen-Morse Non-central Potential Using NU Method and Romanovski Polynomials, J. Phys.: Conf. Series, 423, pp. 012031-1-10, 2013.

[34] Suparmi, A., Cari, C. \& Handhika, J., Approximate Solution of Schrodinger Equation For Eckart Potential Combined With Trigonormetric Pöschl-Teller Non-Central Potential Using Romanovski Polynomials, J. of Phys.: Conf. Series, 423, pp. 012039-1-11, 2013.

[35] Saregar, A., Suparmi, A., Cari, C. \& Yuliani, H., Analysis of Energy Spectra and Wave Function of Trigonometric Pöschl-Teller plus RosenMorse Non-Central Potential Using Supersymmetric Quantum Mechanics Approach, Res. Inv.: Int. J. Eng. and Sci., 2(3), pp. 14-26, 2013.

[36] Suparmi, A., Cari, C., \& Yuliani, H., Energy Spectra and Wave Function Analysis of q-Deformed Modified Pöschl-Teller and Hyperbolic Scarf II Potentials Using NU Method and a Mapping Method, Adv. Phys. Theor. Appl., 16, pp. 64-74, 2013.

[37] Ikhdair, S.M. \& Sever, R., Approximate Bound States of the Dirac Equation with Some physical Quantum Potentials, arXiv:1204.2700v2 [quant-ph], pp. 1-25, 2012

[38] Khare, A. \& Bhaduri, R.K., Supersymmetry Shape Invariance and Exactly Solvable Noncentral Potentials, arXiv:hep-th/9310104v1, pp. 116, 1993.

[39] Cari, Quantum Mechanics-Solutions of Non-Central Potentials Using SUSY Quantum Mechanics, Hypergeometry Method, Nikiforov-Uvarov Method, and Romanovski Polynomial, p. 240, Surakarta UNS Press 2013. 